\title{
Kinetics of a single cross-bridge in familial hypertrophic cardiomyopathy heart muscle measured by reverse Kretschmann fluorescence
}

\section{Prasad Mettikolla \\ Nils Calander \\ Rafal Luchowski \\ Ignacy Gryczynski \\ Zygmunt Gryczynski \\ Julian Borejdo}

University of North Texas Health Science Center Department of Molecular Biology and Immunology Center for Commercialization of Fluorescence Technology

3500 Camp Bowie Boulevard

Fort Worth, Texas 76107

\begin{abstract}
Familial hypertrophic cardiomyopathy (FHC) is a serious heart disease that often leads to a sudden cardiac death of young athletes. It is believed that the alteration of the kinetics of interaction between actin and myosin causes FHC by making the heart to pump blood inefficiently. We set out to check this hypothesis ex vivo. During contraction of heart muscle, a myosin cross-bridge imparts periodic force impulses to actin. The impulses are analyzed by fluorescence correlation spectroscopy (FCS) of fluorescently labeled actin. To minimize observation volume and background fluorescence, we carry out FCS measurements in surface plasmon coupled emission mode in a reverse Kretschmann configuration. Fluorescence is a result of near-field coupling of fluorophores excited in the vicinity of the metal-coated surface of a coverslip with the surface plasmons propagating in the metal. Surface plasmons decouple on opposite sides of the metal film and emit in a directional manner as far-field p-polarized radiation. We show that the rate of changes of orientation is significantly faster in contracting cardiac myofibrils of transgenic mice than wild type. These results are consistent with the fact that mutated heart muscle myosin translates actin faster in in vitro motility assays. () 2010 Society of Photo-Optical Instrumentation Engineers. [DOI: $10.1117 / 1.3324871]$
\end{abstract}

Keywords: microscopy; fluorescence spectroscopy; surface plasmons.

Paper 09517R received Nov. 19, 2009; revised manuscript received Jan. 4, 2010; accepted for publication Jan. 6, 2010; published online Mar. 2, 2010.

\section{Introduction}

Familial hypertrophic cardiomyopathy (FHC) is a serious heart disease that often leads to sudden cardiac death (SCD) at a young age. ${ }^{1,2}$ It is an autosomal dominant disease characterized by left ventricular and septal hypertrophy, and by myofibrillar disarray. ${ }^{3}$ One of the most common causes of FHC is single point mutation in a gene that encodes for the ventricular myosin regulatory light chain (RLC). ${ }^{4-10}$ Clinical studies have revealed that one of the mutations- $\mathrm{R} 58 \mathrm{Q}$ mutation in the myosin RLC - is associated with a malignant FHC disease phenotype. ${ }^{8}$

Heart muscle contraction results from periodic interaction of myosin cross-bridges with actin, during which myosin delivers periodic force impulses to the thin filament. Current hypotheses, based on measurements of muscle force development, suggest that FHC mutations cause alteration of kinetics of this interaction, causing the heart to pump blood inefficiently. ${ }^{11}$ However, isometric force is a temporal average of trillions of individual force impulses. ${ }^{12}$ It is impossible to extract kinetic information from steady-state ensemble measurements. Averaging over many molecules will always mask

Address all correspondence to: Julian Borejdo, Department of Molecular Biology, University of North Texas Health Science Center, 3500 Camp Bowie Boulevard, Fort Worth, Texas 76107, Tel: 817-735-2106; Fax: 817-735-2118; E-mail: jborejdo@hsc.unt.edu individual impulses. However, kinetic information can be extracted from stochastic fluctuations of the signal from small ensembles of molecules. For such applications, fluorescence correlation spectroscopy $(\mathrm{FCS})^{13-16}$ is the method of choice. At the same time, it is important to perform experiments in a tissue because excluded volume effects, which come into play at high protein concentration, may play a significant role during muscle contraction. Concentration of actin and myosin in muscle is extremely high. ${ }^{17}$ At high concentrations, the access to proteins may be limited to only small solvents. As a result, proteins in certain regions of a muscle cell may become overhydrated and behave differently than isolated proteins that are normally hydrated. ${ }^{18,19}$

FCS is typically used together with confocal detection. This goes a long way toward achieving requirements of observing small numbers of molecules under ex vivo conditions. However, in confocal detection, the axial dimension of the detection volume is of the order of a few microns, large compared to the axial extent of the attached biomolecule. Moreover, a significant background is contributed by the reflection and scattering of the excitation light from the surface/ interface. Total internal reflection fluorescence $(\mathrm{TIRF})^{20}$ resolved some of these problems and allowed the application to myosin II and V in vitro. ${ }^{21-26}$ It is possible to further decrease

1083-3668/2010/15(1)/017011/9/\$25.00 @ 2010 SPIE 
axial dimension and avoid reflections and scattering by the use of a surface plasmon assisted microscope (SPAM). ${ }^{27}$ SPAM allows observation of a few molecules by producing extremely thin optical sectioning, and by providing excellent background rejection. The axial dimension decrease is a consequence of the fact that the surface plasmon coupled emission (SPCE) takes advantage of the strongly restricted range for near-field interactions. ${ }^{27-29}$ The axial dimension of SPCE is very small $(\sim 50 \mathrm{~nm})$ due to the restricted range of nearfield interactions of excited fluorophores with surface plasmon polaritons in thin metal film. The coupling distance is further reduced at a close proximity (below $10 \mathrm{~nm}$ ) to a surface by fluorescence quenching by a metal. Typically two types of excitation modes are used. Excitation of the sample directly with the laser beam [reverse Kretschmann configuration $(\mathrm{RK})]$ or through the metal layer via surface plasmon evanescent field [Kretschmann configuration (KR)]. KR mode requires very precise incident angle adjustment, but has the advantage that evanescent wave excitation further reduces the depth of the observed layer. The RK configuration has the advantages that it is very simple to implement, that the exciting light need not be angle adjusted or polarized, and that it avoids losses of intensity because it penetrates only one mirrored surface. Both configurations have the advantage that the fluorescence is observed through the metal film. This assures excellent background rejection, because scattered excitation light is unable to penetrate the metal layer and enter the objective. An additional benefit is the fact that fluorophores coupling to surface plasmons in the metal films strongly depend on fluorophore orientation. Emitting dipoles perpendicular to the surface couple very efficiently, resulting in $p$-polarized emission on the other side of the film, and dipoles parallel to the surface do not couple ( $s$-polarized modes are not supported by the surface plasmons). This near-field-based selection will have a bigger effect than typically used photoselection and fluorescence polarization measurements. In this work we used RK configuration.

A classical method of measuring muscle kinetics is to follow the changes of the orientation of the fluorescent dipole of a dye attached to actin or myosin ${ }^{30-36}$ by using fluorescence polarization. We show here that coupling to surface plasmons is more sensitive to dipole orientation and that the resulting directional emission fluctuations are more pronounced. We utilize FCS detection to follow kinetics of subtle conformational changes occurring on FHC point mutation. This is a simple extension of FCS technology that takes advantage of the fact that orientational fluctuations strongly reflect on the observed SPCE signal.

For observing a few molecules, it is preferable to focus on actin, since it can be labeled with a very low concentration of fluorescent phalloidin. Such labeling preserves the regular structure of a myofibril, phalloidin does not alter the enzymatic properties of muscle, ${ }^{37,38}$ and phalloidin labels actin stoichiometrically, which allows strict control of the degree of labeling. Finally, phalloidin attaches to actin noncovalently but strongly. Noncovalent binding is preferable in the case where changes in the orientation of the dipole moment of a probe are measured. Noncovalent bonds involve attachment over large surfaces through electrostatic and hydrogen links. Observing orientation of actin is a valid way of observing interaction with cross-bridges, because it has been known for a long time that actin changes orientation in response to crossbridge binding, ${ }^{38-41}$ and that those changes parallel the changes of orientation of a cross-bridge. ${ }^{42}$ We a present an application of SPCS to clearly show that actin of transgenic heart muscle affected by R58Q mutation in RLC has a different kinetic behavior than actin from a transgenic wild-type heart muscle.

\section{Materials and Methods}

\subsection{Chemicals and Solutions}

Rhodamine-phalloidin (RP) and rhodamine-labeled microspheres (100 nm diam) were from Molecular Probes (Eugene, Oregon). Spheres were supplied at $3.6 \times 10^{13} / \mathrm{mL}$ and used at $100 \times$ dilution. All other chemicals including 1-ethyl-3-(3' -dimethylaminopropyl) carbodiimide (EDC), and dithiotreitol (DTT), were from Sigma (Saint Louis, Missouri). EDTA-rigor solution contained 50-mM KCl, 2-mM EDTA, 1-mM DTT, 10-mM Tris- $\mathrm{HCl}$ buffer $\mathrm{pH}$ 7.5. Ca-rigor solution contained 50-mM KCl, 4-mM $\mathrm{MgCl}_{2}, 0.1-\mathrm{mM} \mathrm{CaCl}_{2}$, 1-mM DTT, 10-mM Tris-HCl buffer $\mathrm{pH}$ 7.5. Mg-rigor solution contained 50-mM KCl, 4-mM $\mathrm{MgCl}_{2}, 1-\mathrm{mM}$ DTT, $10-\mathrm{mM}$ Tris- $\mathrm{HCl}$ buffer $\mathrm{pH}$ 7.5. Contracting solution was the same as Ca-rigor, except that it contained an addition 5-mM ATP.

\subsection{Microscope Slides}

Glass or sapphire microscope slides were covered with metal by vapor deposition by EMF Corporation (Ithaca, New York). A 48-nm layer of gold was deposited on the slides. A 2-nm chromium undercoat was used as an adhesive background. $\mathrm{NA}=1.45$ objective with gold-coated glass slides, and NA $=1.65$ objective with gold-coated sapphire slides were used.

\subsection{Preparation of Myofibrils}

Transgenic mouse hearts were a gift from Szczesna-Cordary from Miami University. After euthanasia, the hearts from 6 to 7-month-old Tg-R58Q and Tg-WT mice were quickly removed and glycerinated as previously described. ${ }^{43,44}$ Myofibrils from right ventricles were prepared from glycerinated fiber bundles stored at $-20{ }^{\circ} \mathrm{C}$ in glycerinating solution, as described in Mettikolla et al. ${ }^{43}$ After application to a coated coverslip, myofibrils were washed with five volumes of the $\mathrm{Ca}^{2+}$-rigor solution. ${ }^{43}$ Myofibrils were freshly prepared for each experiment. Labeled myofibrils $(25 \mu \mathrm{l})$ were applied to a gold-coated coverslip. The sample was left on a coverslip for $3 \mathrm{~min}$ to allow the myofibrils to adhere. The gold-coated cover slip was covered with a $10 \times 10$-mm coverslip to prevent drying.

\subsection{Preparation of Fluospheres}

Orange fluorescent $(540 / 560 \mathrm{~nm})$ carboxylate modified microspheres $0.1 \mu \mathrm{m}$ in diameter (F-8800, lot 91B2-3) were from Invitrogen (Carlsbad, California). Spheres (2\% solids) were diluted 100 times with water to $3.6 \times 10^{11}$ spheres $/ \mathrm{mL}$.

\subsection{Labeling}

$1-\mathrm{mg} / \mathrm{mL}$ myofibrils ( $\sim 4-\mu \mathrm{M}$ actin) were mixed with $10-\mathrm{nM}$ rhodamine-phalloidin $+10-\mu \mathrm{M}$ unlabeled phalloidin. Unlabeled phalloidin was necessary to prevent uneven label- 
ing. If it was not there, sarcomeres closest to the tip of the pipette, which was used to add the label, would have contained more chromophores than sarcomeres further away from the tip. The degree of labeling was $10 \mu \mathrm{M} / 10 \mathrm{nM}=1000$, i.e., on average, 1 actin protomer in $10^{3}$ was fluorescently labeled. Labeled myofibrils were washed with five volumes of the $\mathrm{Ca}^{2+}$-rigor solution by applying the solution to one end of the channel and absorbing with number- 1 filter paper at the other end.

\subsection{Cross-Linking}

To prevent shortening of muscle in contracting solution, myofibrils $(1 \mathrm{mg} / \mathrm{mL})$ were incubated with $20-\mathrm{mM}$ EDC for $10 \mathrm{~min}$ at room temperature according to the procedure of Herrmann et al. ${ }^{45}$ The reaction was stopped by $20-\mathrm{mM}$ DTT. The lack of shortening was checked by comparing the length of a myofibril before and $100 \mathrm{sec}$ after inducing contraction. Within the limits of measuring accuracy on the computer screen $(\sim 1 \%)$, the length always remained unchanged.

\subsection{Data Collection}

The experiments were done using an ISS Alba FCS (ISS, Urbana, Illinois) confocal system coupled to an Olympus IX 71 microscope. A sample was illuminated with a water immersion objective (Zeiss Planapochromat NA=1.2, 40×) and collected with either a high aperture objective (Olympus Plan Apo NA $=1.45, \times 60)$ through oil with refractive index 1.512, or with a very high aperture objective (Olympus Apo, NA $=1.65,100 \times)$ through high refractive index (1.78) liquid (Cargille Laboratories, Ceder Grove, New Jersey). The excitation was by a $532-\mathrm{nm} \mathrm{cw}$ laser. The confocal pinhole was $50 \mu \mathrm{m}$. Fluorescence was collected every $10 \mu \mathrm{s}$. Orthogonally linearly polarized analyzers were placed before avalanche photodiodes (APDs). The laser was polarized vertically (on the microscope stage). Myofibril was also vertical.

\subsection{Data Analysis}

Fluorescence was collected every $10 \mu \mathrm{s}$ for $20 \mathrm{~s}$ (2-M data points). Correlation functions were computed and fitted using ISS Vista version 4.0.29 software. For plotting, the signal was smoothed by binning 1000 data points (final frequency response is $100 \mathrm{~Hz}$ ).

\subsection{Rotation of Rhodamine-Phalloidin on F-Actin}

For quantitative measurement of orientation, it is important to find out whether the bound probe is immobile. This was done by measuring fluorescence anisotropy by the time-domain technique using a FluoTime 200 fluorometer (PicoQuant, Incorporated, Westfield, Massachusetts). Actin (1 $\mu \mathrm{M})$ was labeled by $0.2-\mu \mathrm{M}$ Alexa488 phalloidin. The excitation was by a 475-nm laser pulsed diode, and the observation was through a monochromator at $590 \mathrm{~nm}$ with a supporting 590-nm-long wave pass filter. FWHM of pulse response function was 68 ps (measured by PicoQuant, Incorporated). Time resolution was better than $10 \mathrm{ps}$. The intensity decays were analyzed in terms of a multiexponential model using FluoFit software (PicoQuant, Incorporated). 100\% of the intensity of phalloidin alone (in the absence of actin) decayed with the time constant of $0.519 \mathrm{~ns}$, consistent with rotation of a molecule with $M_{w}$ $=1250$. No independent rotation of rhodamine moiety was observed. The decay of anisotropy of RP coupled to F-actin was best fitted by the two exponents with correlation times of 0.665 and 36.8 ns with the relative contributions of 13.7 and $86.3 \%$, respectively. The short correlation time is due to the rotation of rhodamine-phalloidin, and the long one to the rotation of F-actin oligomers. Thus over $86 \%$ of fluorescent phalloidin is immobilized by F-actin. This is consistent with the fact that probes attached to proteins through interactions that stretch over large surface areas, such as hydrophobic or Van der Waals interactions, are attached more rigidly than probes that are attached by covalent links. We conclude that fluorescent phalloidin is a good dye for monitoring anisotropy of muscle.

\section{Principle of the Method}

We first consider a conventional case [Fig. 1(a)] [the same argument applies when the light is incident at a sample from the bottom, either perpendicularly (confocal) or at a critical angle (TIRF) or SPR angle]. A muscle myofibril rests on a plain glass coverslip. The incident light (green) is incident on a sample from the top and excites red fluorescence of the labeled molecules. Fluorescence is collected by the objective through the immersion oil. However, some fluorophores are excited indirectly, by the light reflected from the surface of a coverslip or scattered by a sample (marked scattered excitation light). This light (far-field fluorescence) is collected by an objective together with the main fluorescent light. To illustrate this effect, skeletal muscle myofibrils were labeled with 100-nM Alexa647-phalloidin (+10- $\mu \mathrm{M}$ unlabeled phalloidin acting as competition for actin) in the presence of the background in the form of $0.5 \mathrm{mM}$ of rhodamine 800 . Figure 1(b) shows that the fluorescence was completely dominated by the background and no image of myofibrils was discernible. Figure 1(c) shows the back focal plane (BFP) image. For the in-focus source, the microscope objective maps ray propagation angles into off-axis radial positions at the BFP. The result of the sine dependence for spherical refracting surfaces is that a ray originating from a source on-axis at the focal plane and propagating at an angle $\theta$ with respect to the axis will cross the objective's BFP at an off-axis radial distance $r=n f \sin \theta$, where $n$ is the refractive index of the medium (1.515) and $f$ is the focal length of the objective. ${ }^{46}$ Figure $1(\mathrm{c})$ reveals that there is a weak peripheral ring, a diffuse interior, and strong central spot. The ring is due to weak coupling to a high refractive index glass, even in the absence of metal, ${ }^{47}$ the diffuse interior is the image, and the central spot arises because a significant amount of exciting light is able to pass to the detector (the detector is looking directly at the laser; all the light impinging on a sample at 0 -deg angle is passing through the center at BFP). The situation is quite different when a sample rests on a coverslip coated with a thin layer of metal [Fig. 1(d)]. The incident light coming from the top through an objective excites the fluorophores in whole sample volume. About $95 \%$ of the beam is reflected by the metal surface and never reaches the detection system. Excited fluorophores only in close proximity to the surface (below $50 \mathrm{~nm}$ ) couple via near-field interactions to induce surface plasmons. Surface plasmons decouple on the other side of the metal film as a directional emission. Fluorophores farther than $100 \mathrm{~nm}$ from the metal surface emit light (far-field radiation), but the me- 

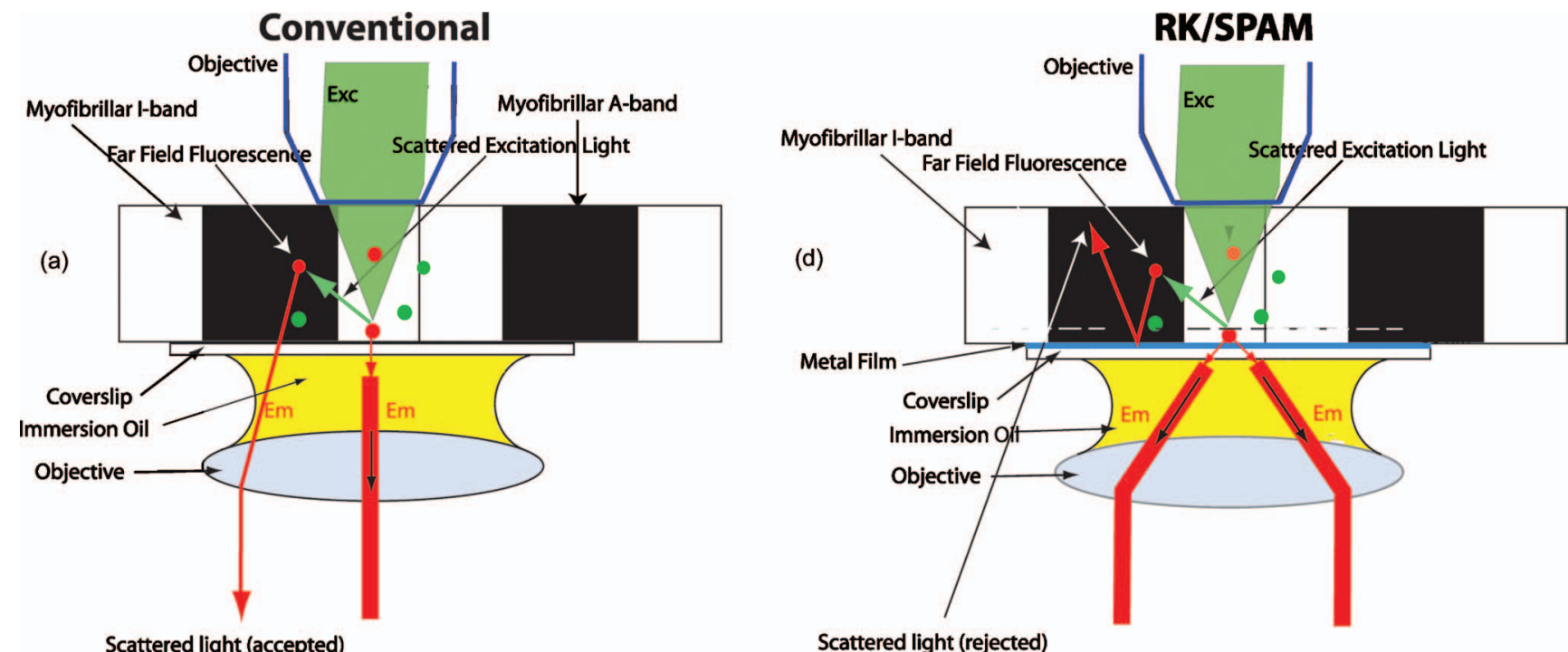

Scattered light (accepted)

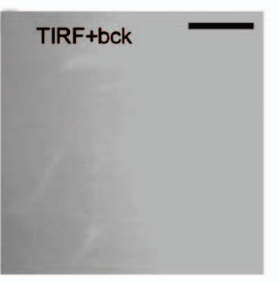

(c)

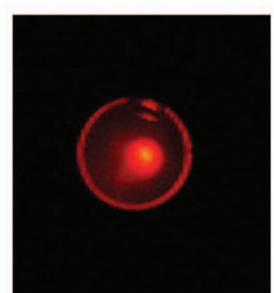

Not excited fluorophores

- Excited fluorophores (e)

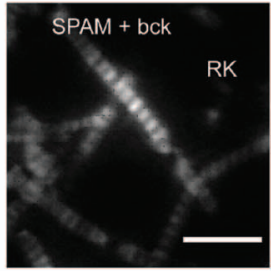

(f)

Fig. 1 Concept of SPAM microscope. A cardiac myofibril is illuminated from above. (a) In a conventional microscope all light, including scattered (background) light, is able to penetrate the coverslip. Green dots represent fluorophores that are out of the field of excitation. Red dots represent fluorophores that are in the path of direct or scattered excitation light. (d) In RK/SPAM, a sample is placed on a metal-coated coverslip and excited with green light (right). The excitation energy from the excited fluorphore couples to the surface plasmons and radiates through the metal film (red) to the objective as a surface of a cone with a half angle equal to the SPCE angle. Metal can be a thin layer of Al (20 nm thick), or Ag or Au (50 nm thick). The scattered light is unable to penetrate the coverslip and is radiated into free space. (b) and (e) The background rejection by SPAM. 0.5-mM rhodamine 800 added as background obscures the image in ordinary TIRF (b). SPAM in the RK configuration eliminates much of the background contribution (e). Myofibrils $(0.1 \mathrm{mg} / \mathrm{mL})$ were labeled with 100-nM Alexa647-+10- $\mu \mathrm{M}$ unlabeled phalloidin for $5 \mathrm{~min}$ at room temperature, then extensively washed with rigor buffer containing 50-mM KCl, 2-mM MgCl, $1-\mathrm{mM} \mathrm{DTT}_{2}$ 10-mM TRIS pH 7.0. 633-nm excitation, 1.65 NA $100 \times$ Olympus objective, sapphire substrate, 1.78 refractive index immersion oil. The bars are $5 \mu \mathrm{m}$ in (b) and $10 \mu \mathrm{m}$ in (e). (c) and (f) The back focal plane image of a sample in a microscope in (c) conventional configuration consists of weak outer ring and a diffuse interior with $s$ strong center corresponding to imperfect blockage of exciting light. The BFP image of a sample in a microscope in ( $\mathrm{f}$ ) RK configuration consists of a strong ring corresponding to emission into free space in a cone.

tallic surface does not transmit it to the detector. Fluorophores closer than $10 \mathrm{~nm}$ to the surface are quenched by the metal. Thus, even though the exciting light does not produce an evanescent wave, the RK/SPAM produces the effect similar to TIRF, because only the fluorescence from molecules within 10 to $50 \mathrm{~nm}$ of the metal layer (indicated by a dashed line) can penetrate the metal layer via plasmon resonance. Moreover, scattered light (far-field fluorescence) is reflected by the metal and is not collected by the objective. When the sample was observed by SPAM in RK configurations, the image was no longer dominated by the background [Fig. 1(e)]. The BFP image of the rhodamine fluorophore on gold-coated glass [Fig. 1(f)] is now doughnut-shaped, because the directional emission emerging from the sample is contained within a cone with a well-defined angle $\Theta$. In conclusion: RK/SPAM provides excellent background rejection, because it combines excitation volume comparable to TIRF with the fact that all the light scattered in a sample is unable to penetrate the metal. ${ }^{48}$

Another characteristic of SPAM is that fluorescence coupling to surface plasmons dramatically depends on the orientation of the fluorescent molecule transition moment. This is illustrated in Fig. 2(a), which compares, for SPCE and TIRF modes of detection, the power entering the microscope objective at different distances from the coverslip for different polar angles of the transition dipole. It is clear that at distances larger than $10 \mathrm{~nm}$, more fluorescence is contributed by SPCE 


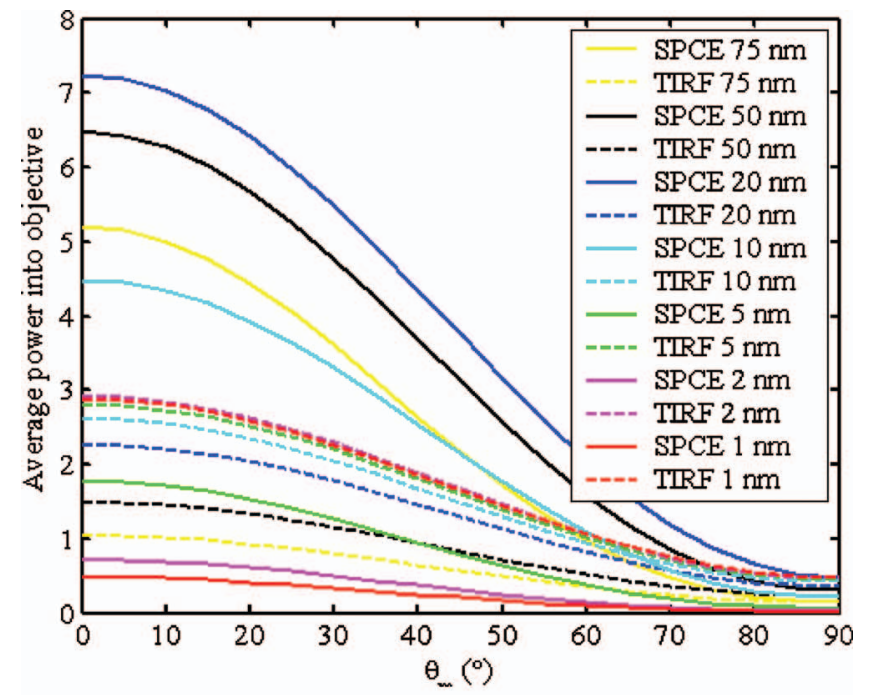

(a)

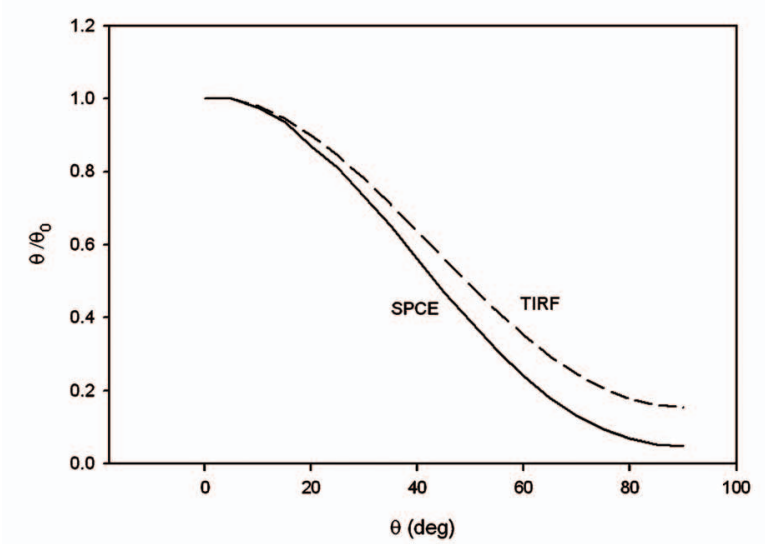

(b)

Fig. 2 (a) Power entering the objective at various distances from the surface of a coverslip. (b) The SPCE (solid line) and TIRF (broken line) power entering the objective at various polar angles. Curves normalized to power at $\theta=0 \mathrm{deg}$.

than TIRF. Not only is the power entering the objective higher for SPCE, but it is more sensitive than TIRF to changes in polar angle. Figure 2(b) compares the sensitivity of SPCE versus TIRF to change in angle. This makes the SPCE method particularly suited to the measurements of protein orientation changes. This is the method used here, i.e., fluctuations arise from changes in the degree of coupling as the dipole changes orientation. This is different than a classical method of measuring orientation changes, which follows anisotropy of a fluorescent dipole of a dye attached to a molecule of interest. $^{49}$

\section{Results}

\section{Photon Flux from a Single Chromophore.}

To estimate this number, we measured signal from a freely diffusing chromophore (the same as labeling muscle) as the absence of photobleaching. We used 50-nM rhodamine, which translates to an average of 100 molecules in the 3-fL detection volume (DV) at any time. No overall bleaching oc-

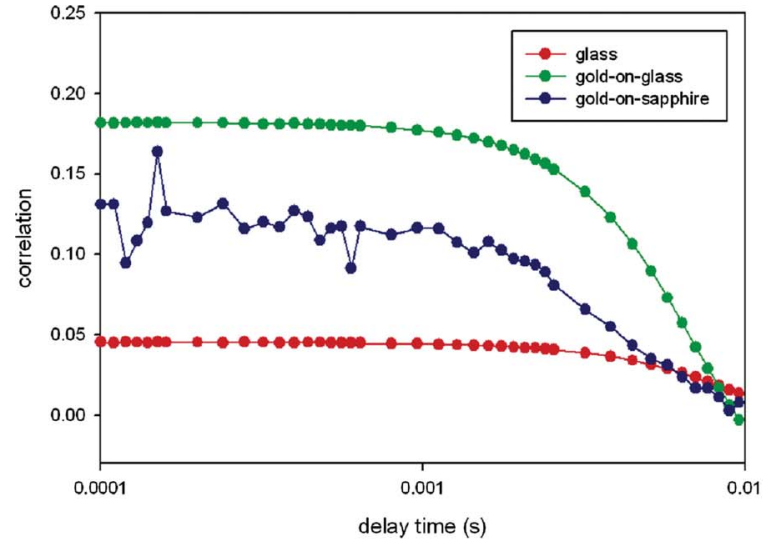

(a)

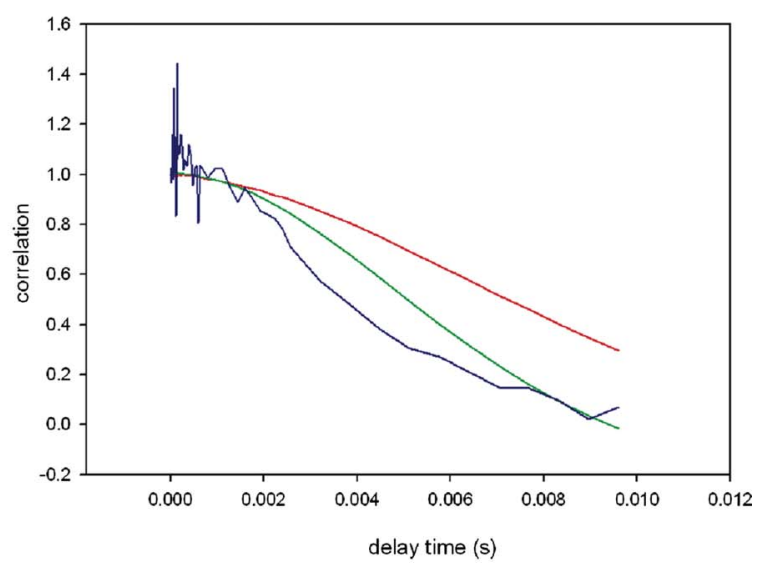

(b)

Fig. 3 RK-SPAM FCS experiments using fluorescent spheres diffusing on glass, gold-coated glass, and gold-coated sapphire coverslips. (a) Correlation function of spheres diffusing on glass (red), gold-coated glass (green), and gold-coated sapphire (blue). The average number of spheres in DV was 6, 8, and 22, respectively. (b) The decay of the correlation function is fastest for spheres diffusing on gold-coated sapphire and slowest for glass. The total photon rate was $\approx 600 \mathrm{Kcounts} / \mathrm{s}$. The incident power is $150 \mu \mathrm{W}$. (Color online only.)

curs because fluorophores spend only limited amounts of time in the DV. These 100 molecules gave parallel $\left(I_{H}\right)$ and perpendicular $\left(I_{V}\right)$ intensities on gold of $I_{H}=7125 \pm 1040$ and $I_{V}=6442 \pm 1721$ photons $/ 10 \mathrm{~ms} 10 \mathrm{~ms}$ for the total photon rate $I_{\text {tot }}=20407$ photons $/ 10 \mathrm{~ms}$. Therefore, the average number of observed photons per fluorophore was $\sim 200 / 10 \mathrm{~ms}$.

\subsection{Polystyrene Spheres}

To show that the method is superior to conventional confocal techniques of measuring motions of protein-sized particles, we compared Brownian motion of $100-\mathrm{nm}$ polystyrene spheres. Figure 3 compares typical RK-SPAM FCS experiments of $0.1-\mu \mathrm{m}$ fluorospheres diffusing on glass goldcoated-glass, and gold-coated-sapphire coverslips. The average counts were $I_{H}=1746 \pm 694$ and $I_{V}=2189 \pm 785$ photons $/ 10 \mathrm{~ms}$, respectively. The total photon rate was $I_{\text {tot }}$ $=I_{V}+2 * G^{*} I_{H}=5681$ photons $/ 10 \mathrm{~ms} \approx 600 \mathrm{Kcounts} / \mathrm{s}$, where $\mathrm{G}$ is the correction factor (0.98). Figure 3(a) compares the correlation function of spheres diffusing on glass (red), goldcoated glass (green), and gold-coated sapphire (blue). For 
spheres diffusing on glass, the magnitude of the correlation function at zero delay time is equal to the inverse of the number of particles in the detection volume, and gives $\sim 22$ spheres in the DV. Since the confocal volume is $\sim 3 \mathrm{fL}$, the concentration of spheres is $7 \times 10^{12} / \mathrm{mL}$, close to the number estimated by Molecular Probes (Invitrogen, Life Technologies, Carlsbad, California) $(100 \times$ dilution of the stock concentration of $3.6 \times 10^{13} / \mathrm{mL}$ ). [Incidentally, it is possible to calculate the number of dyes per $0.1-\mu \mathrm{m}$ FluoSphere: the dye contained in Invitrogen orange fluorescent carboxylate modified FluoSpheres is proprietary. We assume that it is Alexa555, because the emission spectrum of spheres is identical to that of the oligo conjugate of Alexa555. Experiments (not shown) suggest that the emission spectra of $0.1 \mu \mathrm{M}$ of $\mathrm{Al}-$ exa555 dye with 200 times dilution of spheres (1.8 $\times 10^{11}$ spheres $\left./ \mathrm{mL}\right)$. $0.1-\mu \mathrm{M}$ dye contains 600 $\times 10^{11}$ molecules $/ \mathrm{mL}$. Since the suspension of spheres fluoresces 22 times as bright as the dye, yet it contains 600/1.8 $=333$ times smaller number of particles, each sphere behaves as if it contained $22 \times 333 \approx 7300$ dye molecules.] Figure 3(a) shows also that the magnitude of correlation function at zero delay time of spheres diffusing on gold-coated glass was nearly four times larger than for spheres diffusing on glass. This is consistent with the fact that gold coating decreases DV by quenching fluorescence near the metal surface. ${ }^{50}$ Figure 3(b) shows that the decay of the correlation function is fastest for spheres diffusing on gold-coated sapphire and slowest for glass. This is due to the fact that experiments on sapphire were carried out using a high aperture objective $(\mathrm{NA}=1.65)$, which has the thinnest depth of focus, and the fact that gold coating decreases DV. Since the correlation function was noisy for gold-coated sapphire, we used gold-coated glass for the rest of the experiments.

The decay of correlation occurs between $10^{-3}$ and $10^{-2} \mathrm{~s}$, consistent with earlier results obtained for a $100-\mathrm{nm}$-diam sphere with a diffusion coefficient of $4.12 \times 10^{-12} \mathrm{~m}^{2} / \mathrm{s}^{51}$ diffusing along the $z$ axis through a distance of $36 \mathrm{~nm}^{48}$

\subsection{Contracting Heart Muscle}

The myofibril was labeled with $10-n M ~ R P+10-\mu M$ UP. Thus only one in 1000 actin monomers carried fluorescent phalloidin. The length, width, and height of a typical half sarcomere (HS) are 1, 1, and $0.5 \mu \mathrm{m}$, respectively, and therefore its volume is $5 \times 10^{-16} \mathrm{~L}$. Since the concentration of actin in muscle is $0.6 \mathrm{mM},{ }^{17}$ this volume contains $\sim 200,000$ actin monomers. Therefore, there are $\sim 200$ fluorophores per HS. The diameter of DV $(0.9 \mu \mathrm{m})$ is equal to the diameter of the confocal pinhole $(50 \mu \mathrm{m})$ divided by the magnification of the objective $(60 \times)$. Therefore, the objective captures entire fluorescence from one HS (200 fluorophores). Since the signal from a freely diffusing chromophore is 200 photons $/ 10 \mathrm{~ms}$, in the absence of photobleaching we would have observed 200 $\times 200=40-K$ counts $/ 10 \mathrm{~ms}$. In fact, the signal [Fig. 4(a)] was many folds smaller: the average counts on gold were $I_{V}$ $=28 \pm 8$ and $I_{H}=72 \pm 17$ photons $/ 10 \mathrm{~ms}$ for the total photon rate $I_{\text {tot }}=174$ photons $/ 10 \mathrm{~ms}$. This shows that photobleaching is a significant factor in our experiments. In contrast to free diffusion, where fluorophores spend only $\mu$ seconds in DV, in our experiments muscle is immobilized on a coverslip and each fluorophore is exposed to light for the few minutes re-

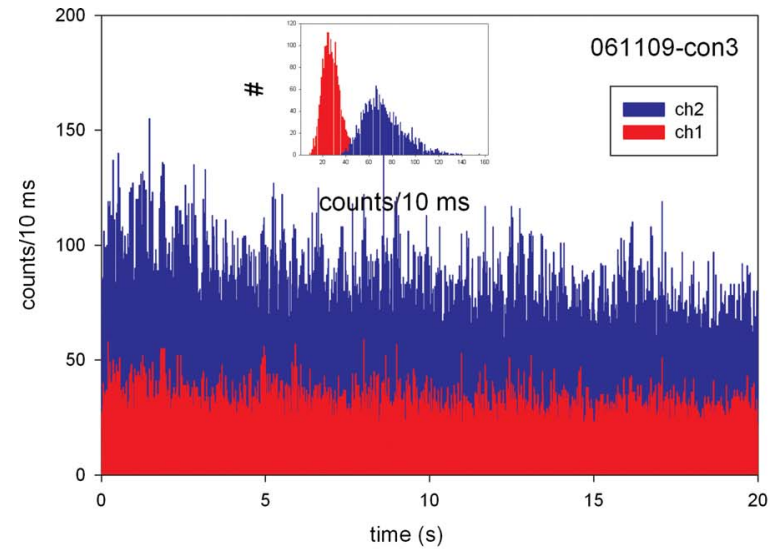

(a)

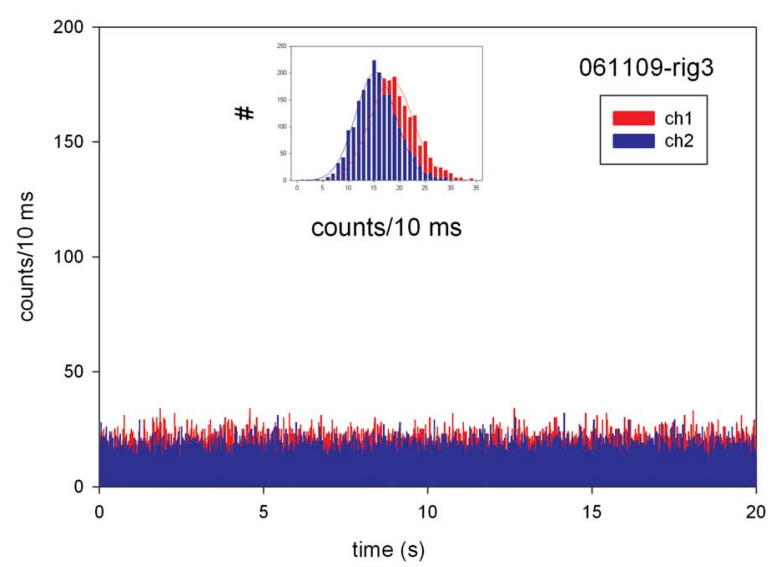

(b)

Fig. 4 Time traces from the left ventricle on gold-coated glass labeled with $10-\mathrm{nM}$ rhodamine phalloidin $+10-\mu \mathrm{M}$ unlabeled phalloidin. (a) Contracting and (b) rigor muscle. Parallel $\left(I_{H}\right)$ and perpendicular $\left(I_{V}\right)$ intensities are shown in the blue and red, respectively. Insets show histograms of counts during (a) contraction and (b) rigor. Note that the Gaussian curve used to fit the contraction data is asymmetrical. (Color online only.)

quired to focus and measure. This causes bleaching of most of the fluorophores in muscle, and by the time the measurement is taken, only the few remaining ones give rise to the observed signal. Figure 4(b) is the control intensity trace of muscle in rigor, when no mechanical activity occurs.

A histogram of photocounts during contraction and rigor is shown in the insets of Figs. 4(a) and 4(b), respectively. In all experiments the Gaussian curve used to fit the data was asymmetrical during contraction. In the example shown in the inset for Fig. 4, the fit to the function $f=\operatorname{aexp}\left\{-.5\left[\left(\mathrm{x}-\mathrm{x}_{\mathrm{o}}\right) / b\right]^{2}\right\}$ gave $R^{2}=0.985$ for ch2 during rigor and $R^{2}=0.922$ for ch2 during contraction. The contraction data also failed a normality test (test failed with $P=0.0007$ ). The normality test checks whether the data passed or failed the test of the assumption that the source population is normally distributed around the regression, and the $P$ value calculated by the test. The marked skewness and kurtosis of polarized intensity during contraction is probably due to the fact that a cross-bridge power stroke changes the orientation in two or more steps, as originally proposed by Huxley and Simmons. ${ }^{52}$ Rigor data, in contrast, passed the test with $P=0.3755$. Failure of the normality 


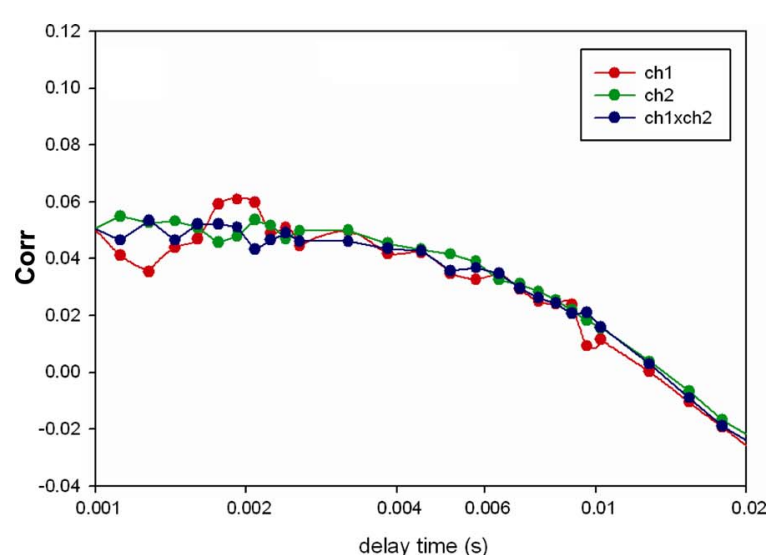

(a)

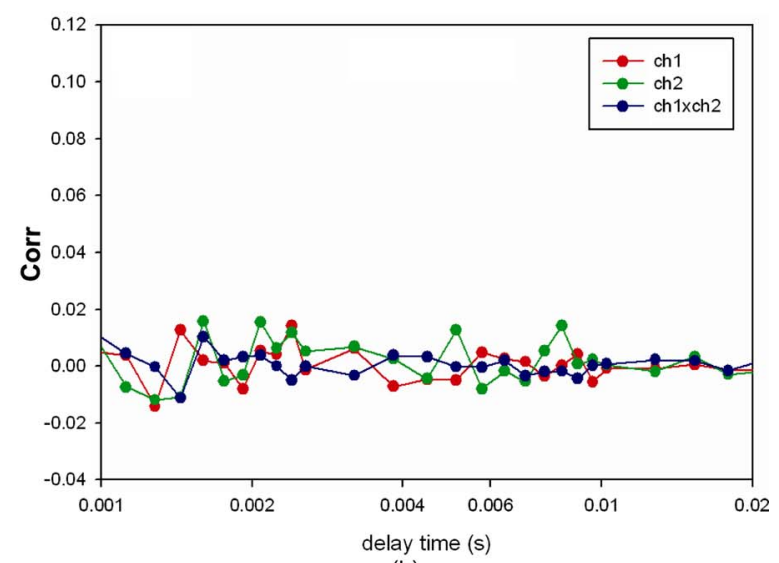

(b)

Fig. 5 Correlation function of Tg-R58Q myofibrils. (a) Contracting myofibrils. (b) Rigor myofibrils. red-autocorrelation function of ch1 $\left(I_{H}\right)$, green-ch2 $\left(I_{V}\right)$, blue-cross-correlation ch1 $\times$ ch2. (Color online only.)

test can indicate the presence of outlying influential points or an incorrect regression model. This may indicate that contraction is a multistep process, as originally proposed. ${ }^{52}$

The correlation functions computed from the signal shown in Fig. 4 is plotted in Fig. 5. The expression for rotational autocorrelation function is very complex. Even the simplest case-rotational diffusion on glass-depends on the angle between the excitation and emission transition dipole moments, the polarization of the excitation, and the presence of emission polarizers. In the simplest case, where the molecule does not rotate during the excited state lifetime, the excitation and emission transition dipole moments are parallel, muscle is excited with polarized light and the emission is observed without polarizers, and the complex expression for the rotational correlation function assumes exponential form $G(\tau)$ $=B \exp \left(-\tau / \tau_{r}\right)$, where $B$ is a constant and $\tau_{r}$ is the rotational correlation time. ${ }^{53}$ The excited state lifetime of rhodamine is $4.08 \mathrm{~ns},{ }^{54}$ and during such a short time actin filament does not rotate to any significant extent. Since the excitation and emission transition dipole moments of rhodamine are almost parallel above $470 \mathrm{~nm}$, the decay of the correlation function is well described by a simple exponential. Pre-exponential constant $B$ is taken as a measure reflecting kinetics of contracting heart muscle. While this scheme is an oversimplification of

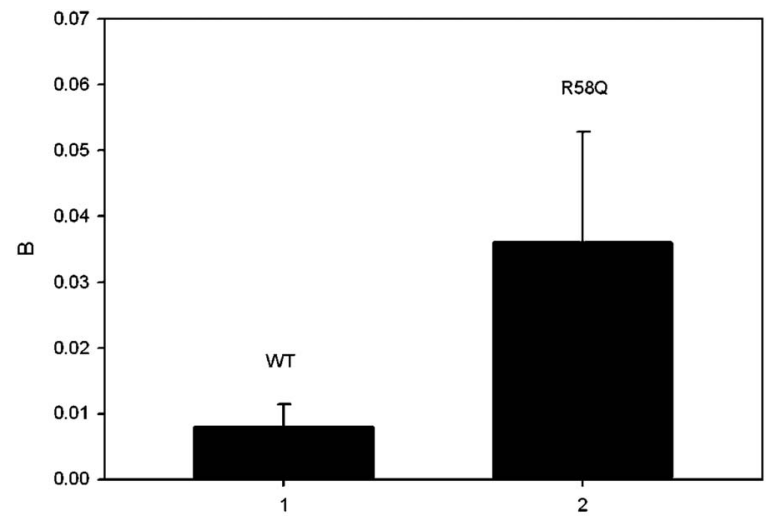

Fig. 6 Pre-exponential constant B from a fit of WT (left) and mutated (right) myofibrils. The error bar is $\mathrm{SD} . \mathrm{N}=5$ for $\mathrm{R} 58 \mathrm{Q}, 4$ for $\mathrm{WT}$.

the actual events (see the discussion in Sec. 5), the constant B reflects a clear difference between WT and mutated muscle. The experiment was repeated four times for $\mathrm{Tg}$-WT and five times for Tg-mutated hearts. Figure 6 summarizes the results and shows that there is a statistically significant $(t=-3.16$, $P=0.034$, paired-test) difference between WT and mutated myofibrils. The correlation function of the rigor control shows no decay at all [Fig. 5(b)].

\section{Discussion}

SPAM can be built in two configurations: reverse Kretschmann (RK) configuration as described before, when the laser beam strikes a cell directly, and in Kretschmann configuration (KR), where the laser beam strikes a sample from below at SPR angle. RK has the advantages that it avoids losses of intensity due to penetration of mirrored surface, it avoids losses inherent in a dichroic mirror, and it is simpler and less expensive to implement. (KR has the advantage that SPR illumination excites evanescent waves at the glass-buffer interface. This makes the optical sectioning thinner, because it is now a product of two near-field factors: the depth of evanescent wave excitation and a distance-dependent coupling of excited fluorophores to the surface plasmons.) The RK/SPAM method has three additional characteristic features that make it particularly suitable for FCS. 1. It has excellent background rejection, made possible by the fact that scattered excitation light is unable to penetrate the metal layer and enter the objective (Fig. 1). In SPAM, the scattered excitation light, which is a predominant problem in FCS measurements in tissue, is unable to penetrate the metal-coated coverslip that acts as a simple mirror. In conventional detection, however, scattered light has no difficulty penetrating the coverslip and entering the objective. 2. The coupling of the fluorescence is strongly distance dependent and extends only to about $50 \mathrm{~nm}$ into a sample, smaller than the 100 to $200 \mathrm{~nm}$ characteristic of TIRF (Fig. 2). It is further reduced at close proximity (below $10 \mathrm{~nm}$ ) to a surface by quenching by a metal. Thus using SPAM, detection of signal is confined to a window of $10 \mathrm{~nm}$ above the surface to $50 \mathrm{~nm}$ above the surface of the metal plate. This property of only detecting light that originates from this $40-\mathrm{nm}$-wide window is a main factor in reducing the background signal. In effect, RK-SPAM is 
equivalent to TIRF excitation without the need to produce evanescent wave. 3. Fluorescence coupling to surface plasmons dramatically depends on the orientation of the molecule transition moment, i.e., the method is particularly suited to measurements of macromolecular orientation changes. This feature has been exploited here.

In addition, it is important to note that coupling preserves very well the spectral properties of fluorophores. ${ }^{55-57}$

We applied RM/SPAM to study an important biological problem-familial hypertrophic cardiomyopathy (FHC). It is a serious heart disease that often leads to a sudden cardiac death. ${ }^{1,2}$ The disease is associated with single-point mutations that occur in sarcomeric proteins. We are interested specifically in mutations in genes that encode for the ventricular myosin regulatory light chain (RLC). ${ }^{4-10}$ In particular, we studied mutation R58Q in the myosin RLC. Heart muscle contraction results from the periodic interaction of myosin cross-bridges with actin, during which myosin delivers periodic force impulses to the thin filament. It is believed that FHC mutations cause alteration of kinetics of this interaction, causing the heart to pump blood inefficiently. ${ }^{11}$ We used RKSPCE to compare the kinetics of interaction of actin with myosin in myofibrils prepared from $\mathrm{Tg}-\mathrm{WT}$ and $\mathrm{Tg}-\mathrm{R} 58 \mathrm{Q}$ hearts. The signals were noisy, because they were contributed by a small fraction of $\sim 200,000$ actins present in a halfsarcomere. The question arises of what kinetic parameter does the FCS spectrum represent? In FHC muscle carrying D166V mutation in RLC, the fluctuations were well described by ONOFF binding and dissociation of a myosin cross-bridge. ${ }^{58}$ Anisotropy of actin was high when it was immobilized by binding to myosin and low when it was free (detached) from myosin. A signal was approximated by a rectangular wave, a "high" of a wave corresponding to a high anisotropy of actin when it was immobilized by binding to myosin, and "low" by low anisotropy when it was free (detached) from myosin. The corresponding correlation function was a series of triangles that were well reproduced by an experiment. In contrast, the correlation function of R58Q muscles analyzed here was not a series of triangles. It was best approximated by a single exponential function. The kinetic parameter extracted from the correlation spectra was the amplitude of the correlation function. This parameter showed statistically significants differences between Tg-WT and Tg-mutated heart muscle. We recognize that fitting our data to simple exponential decay is a gross oversimplification of the actual events. The orientation change of myosin cross-bridges, and therefore of actin, does not occur in a single reversible step, but as suggested by asymmetry of the histogram shown in the inset in Fig. 4(a), it probably occurs in a series of smaller steps. Moreover, it is reversed in a series of mechanical steps, each associated with definite biochemical transition. ${ }^{59,60}$ Finally, some rotation of the rhodamine moiety does occur during the excited state lifetime (unpublished data), and the emission is observed through polarizers. Nevertheless, exponential kinetics effectively reflect the difference in kinetics of healthy and diseased heart. The fact that the amplitude is increased in mutated muscle is consistent with the fact that the ATPase rate and the maximal velocity of actin translation in the in vitro motility assay was larger for R58Q myosin in comparison with WT myosin. ${ }^{61} \mathrm{At}$ the same time, maximal force developed by mutated muscle was not increased, suggesting a decrease in the efficiency of ATP utilization. Thus the present work is consistent with the idea that FHC results from decreased efficiency.

The diagnosis of the disease in human patients is made more complex by the fact that humans are normally heterozygous for FHC disease. Consequently, 50\% of their myosincontaining thick filaments are composed of non mutant myosin heads interspersed with FHC mutant heads. We therefore anticipate that $50 \%$ of experiments using human tissue would exhibit R58Q-mediated cross-bridge kinetics, and 50\% of experiments would exhibit WT-mediated cross-bridge kinetics. (Transgenic mouse hearts used here, in contrast, express R58Q mutation in nearly $100 \%$ of cases.) On the other hand, diagnosis in humans would be made easier by the fact that MYL2 gene expression occurs both in the heart and in the slow skeletal muscle. The biopsies for diagnosis could be therefore obtained from the soleus muscle of the R58Q patients. This could be advantageous, not only because biopsied samples are easy to obtain, but also because the regular sarcomere organization of the skeletal muscle myofibrils make the experiments easier to perform.

\section{Conclusions}

We observe polarized fluorescence from actin molecules in working mouse hearts suffering from FHC. The use of a surface plasmon coupled emission microscope in a reverse Kretschmann configuration minimizes observation volume, background fluorescence, and scattering. The correlation function of R58Q muscles is approximated by a single exponential function. Its amplitude shows statistically significant differences between Tg-WT and Tg-mutated heart muscle.

\section{Acknowledgments}

Supported by NIH grants R01AR048622 and R01 HL090786 to Borejdo, and by Texas ETF grant (CCFT).

\section{References}

1. B. J. Maron, "The young competitive athlete with cardiovascular abnormalities: causes of sudden death, detection by preparticipation screening, and standards for disqualification," Card. Electrophysiol. Rev. 6, 100-103 (2002).

2. B. J. Maron, I. Olivotto, P. Spirito, S. A. Casey, P. Bellone, and T. E. Gohman, "Epidemiology of hypertrophic cardiomyopathy-related death: revisited in a large non-referral-based patient population," Circulation 102, 858-864 (2000).

3. L. L. Tin, D. G. Beevers, and G. Y. Lip, "Hypertension, left ventricular hypertrophy, and sudden death," Curr. Cardiol. Rep. 4(6), 449457 (2002).

4. K. Poetter et al., "Mutations in either the essential or regulatory light chains of myosin are associated with a rare myopathy in human heart and skeletal muscle," Nat. Genet. 13(1), 63-69 (1996).

5. J. Flavigny et al., "Identification of two novel mutations in the ventricular regulatory myosin light chain gene (MYL2) associated with familial and classical forms of hypertrophic cardiomyopathy," J. Mol. Med. 76(3-4), 208-214 (1998).

6. P. S. Andersen et al., "Myosin light chain mutations in familial hypertrophic cardiomyopathy: phenotypic presentation and frequency in Danish and South African populations," J. Med. Genet. 38(12), E43 (2001).

7. Z. T. Kabaeva et al., "Systematic analysis of the regulatory and essential myosin light chain genes: genetic variants and mutations in hypertrophic cardiomyopathy," Eur. J. Hum. Genet. 10(11), 741-748 (2002).

8. P. Richard et al., "Hypertrophic cardiomyopathy: distribution of disease genes, spectrum of mutations, and implications for a molecular 
diagnosis strategy," Circulation 107, 2227-2232 (2003).

9. S. Morner et al., "Identification of the genotypes causing hypertrophic cardiomyopathy in northern Sweden," J. Mol. Cell. Cardiol. 35(7), 841-849 (2003).

10. L. Hougs et al., "One third of Danish hypertrophic card iomyopathy patients have mutations in MYH7 rod region," Eur. J. Hum. Genet. 13, 161-165 (2005).

11. W. G. Kerrick et al., "Malignant familial hypertrophic cardiomyopathy D166V mutation in the ventricular myosin regulatory light chain causes profound effects in skinned and intact papillary muscle fibers from transgenic mice," FASEB J. 23(3), 855-865 (2009).

12. A. Oplatka, "On the mechanochemistry of muscular contraction," $J$. Theor. Biol. 34(2), 379-403 (1972).

13. D. Magde, E. L. Elson, and W. W. Webb, "Fluorescence correlation spectroscopy II. An experimental realization," Biopolymers 13(1), 29-61 (1974).

14. E. L. Elson and D. Magde, "Fluorescence correlation spectroscopy: coceptual basis and theory," Dev. Mech. 13, 1-28 (1974).

15. E. L. Elson, "Fluorescence correlataion spectroscopy and photobleaching recovery," Аnnu. Rev. Phys. Chem. 36, 379-406 (1985)

16. E. L. Elson, "Quick tour of fluorescence correlation spectroscopy from its inception," J. Biomed. Opt. 9(5), 857-864 (2004).

17. C. R. Bagshaw, Muscle Contraction, Chapman and Hall, London (1982).

18. A. P. Minton and J. Wilf, "Effect of macromolecular crowding upon the structure and function of an enzyme: glyceraldehyde-3-phosphate dehydrogenase," Biochemistry 20(17), 4821-4826 (1981).

19. A. P. Minton, "Excluded volume as a determinant of macromolecular structure and reactivity," Biopolymers 20, 2093-2120 (1981).

20. D. Axelrod, "Total internal reflection fluorescence microscopy in cell biology," Traffic 2, 764-774 (2001).

21. D. M. Warshaw et al., "Myosin conformational states determined by single fluorophore polarization," Proc. Natl. Acad. Sci. U.S.A. 95(14), 8034-8039 (1998).

22. M. E. Quinlan, J. N. Forkey, and Y. E. Goldman, "Orientation of the myosin light chain region by single molecule total internal reflection fluorescence polarization microscopy," Biophys. J. 89(2), 1132-1142 (2005).

23. J. Enderlein and W. P. Ambrose, "Optical collection efficiency function in single-molecule detection experiments," Appl. Opt. 36(22), 5298-5302 (1997).

24. K. A. Willets et al., "Novel fluorophores for single-molecule imaging," J. Am. Chem. Soc. 125(5), 1174-1175 (2003).

25. Y. Wang et al., "Single-molecule structural dynamics of EF-Gribosome interaction during translocation," Biochemistry 46(38), 10767-10775 (2007).

26. Y. Taniguchi et al., "Single molecule thermodynamics in biological motors," BioSystems 88(3), 283-292 (2007).

27. J. Borejdo et al., "Application of surface plasmon coupled emission to study of muscle," Biophys. J. 91, 2626-2635 (2006).

28. I. Gryczynski, J. Malicka, Z. Gryczynski, and J. R. Lakowicz, "Surface plasmon-coupled emission with gold films," J. Phys. Chem. B 108, 12568-12574 (2004)

29. I. Gryczynski, J. Malicka, J. R. Lakowicz, E. M. Goldys, N. Calendar, and Z. Gryczynski, "Two-photon induced surface plasmoncoupled emission," Thin Solid Films 491, 173-176 (2005).

30. C. G. Dos Remedios, R. G. Millikan, and M. F. Morales, "Polarization of tryptophan fluorescence from single striated muscle fibers. A molecular probe of contractile state," J. Gen. Physiol. 59, 103-120 (1972).

31. C. G. Dos Remedios, R. G. Yount, and M. F. Morales, "Individual states in the cycle of muscle contraction," Proc. Natl. Acad. Sci. U.S.A. 69, 2542-2546 (1972).

32. T. Nihei, R. A. Mendelson, and J. Botts, "Use of fluorescence polarization to observe changes in attitude of S1 moieties in muscle fibers," Biophys. J. 14, 236-242 (1974).

33. R. T. Tregear and R. A. Mendelson, "Polarization from a helix of fluorophores and its relation to that obtained from muscle," Biophys. J. 15, 455-467 (1975).

34. D. D. Thomas and R. Cooke, "Orientation of spin-labeled myosin heads in glycerinated muscle fibers," Biophys. J. 32, 891-905 (1980).

35. J. Borejdo et al., "Cross-bridge orientation in skeletal muscle measured by linear dichroism of an extrinsic chromophore," J. Mol. Biol. 158, 391-414 (1982).
36. M. F. Morales, "Calculation of the polarized fluorescence from a labeled muscle fiber," Proc. Natl. Acad. Sci. U.S.A. 81, 145-149 (1984).

37. A. E. Bukatina, F. Fuchs, and S. C. Watkins, "A study on the mechanism of phalloidin-induced tension changes in skinned rabbit psoas muscle fibres," J. Muscle Res. Cell Motil. 17(3), 365-371 (1996).

38. E. Prochniewicz-Nakayama, T. Yanagida, and F. Oosawa, "Studies on conformation of F-actin in muscle fibers in the relaxed state, rigor, and during contraction using fluorescent phalloidin," J. Cell Biol. 97, 1663-1667 (1983).

39. T. Yanagida and F. Oosawa, "Conformational changes of F-actinepsilon-ADP in thin filaments in myosin-free muscle fibers induced by Ca2+," J. Mol. Biol. 140(2), 313-320 (1980).

40. T. Yanagida and F. Oosawa, "Polarized fluorescence from epsilonADP incorporated into F-actin in a myosin-free single fiber, conformation of F-actin and changes induced in it by heavy meromyosin," J. Mol. Biol. 126(3), 507-524 (1978).

41. Y. S. Borovikov, N. V. Kuleva, and M. I. Khoroshev, "Polarization microfluorimetry study of interaction between myosin head and F-actin in muscle fibers," Gen. Physiol. Biophys. 10(5), 441-459 (1991).

42. J. Borejdo et al., "Changes in orientation of actin during contraction of muscle," Biophys. J. 86, 2308-2317 (2004).

43. P. Mettikolla, R. Luchowski, I. Gryczynski, Z. Gryczynski, D. Szczesna-Cordary, and J. Borejdo, "Fluorescence lifetime of actin in the FHC transgenic heart," Biochemistry 48, 1264-1271 (2009).

44. D. Szczesna-Cordary et al., "The E22K mutation in myosin RLC that causes familial hypertrophic cardiomyopathy increases calcium sensitivity of force and ATPase in transgenic mice," J. Cell. Sci. 118 , 3675-3683 (2005).

45. C. Herrmann et al., "A structural and kinetic study on myofibrils prevented from shortening by chemical cross-linking," Biochemistry 32(28), 7255-7263 (1993).

46. A. L. Mattheyses and D. Axelrod, "Fluorescence emission patterns near glass and metal-coated surfaces investigated with back focal plane imaging," J. Biomed. Opt. 10(5), 054007 (2005).

47. J. Enderlein and T. Ruckstuhl, "The efficiency of surface-plasmon coupled emission for sensitive fluorescence detection," Opt. Express 13(22), 8855-8865 (2005).

48. J. Borejdo et al., "Fluorescence correlation spectroscopy in surface plasmon coupled emission microscope," Opt. Express 14(17), 78787888 (2006).

49. A. J. Pesce, C. G. Rosen, and T. L. Pasby, Fluorescence Spectroscopy, Marcel Dekker, New York (1971).

50. N. Calander et al., "Fluorescence correlation spectroscopy in a reverse Kretchmann surface plasmon assisted microscope," Opt. Express 16(17), 13381-13390 (2008).

51. C. Tanford, Physical Chemistry of Macromolecules, John Wiley and Sons, New York (1963).

52. A. F. Huxley and R. M. Simmons, "Proposed mechanism of force generation in striated muscle," Nature 233, 533-538 (1971).

53. M. Ehrenberg and R. Rigler, "Fluorescence correlation spectroscopy applied to rotational diffusion of macromolecules," Q. Rev. Biophys. 9(1), 69-81 (1976).

54. ISS, "Lifetime data of selected fluorophores," see http:// www.iss.com/resources/fluorophores.html (2008).

55. E. Matveeva et al., "Myoglobin immunoassay utilizing directional surface plasmon-coupled emission," Anal. Chem. 76, 6287-6292 (2004).

56. J. Malicka et al., "Surface plasmon-coupled ultraviolet emission of 2,5-diphenyl-1,3,4-oxadiazole," J. Phys. Chem. B 108, 19114-19118 (2004).

57. I. Gryczynski et al., "Surface plasmon-coupled emission of quantum dots," J. Phys. Chem. B 109, 1088-1093 (2005).

58. P. Muthu et al., "Single molecule kinetics in the familial hypertrophic cardiomyopathy D166V mutant mouse heart," J. Mol. Cell. Cardiol. (in press).

59. A. Houdusse and H. L. Sweeney, "Myosin motors: missing structures and hidden springs," Curr. Opin. Struct. Biol. 11(2), 182-194 (2001).

60. M. A. Geeves and K. C. Holmes, "The molecular mechanism of muscle contraction," Adv. Protein Chem. 71(24), 161-193 (2005).

61. M. J. Greenberg et al., "Regulatory light chain mutations associated with cardiomyopathy affect myosin mechanics and kinetics," J. Mol. Cell. Cardiol. 46(1), 108-115 (2009). 